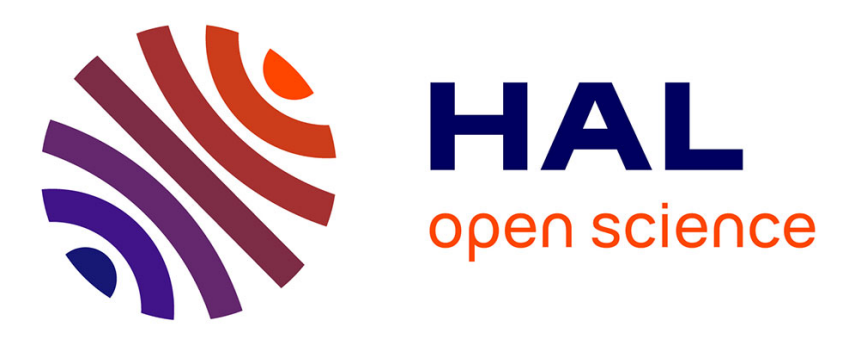

\title{
Topology Optimization of a Reactionless Four-bar Linkage
}

Sébastien Briot, Alexandre Goldsztejn

\section{To cite this version:}

Sébastien Briot, Alexandre Goldsztejn. Topology Optimization of a Reactionless Four-bar Linkage. 7th IFToMM International Workshop on Computational Kinematics (CK2017), May 2017, Poitiers, France. hal-01479410

\author{
HAL Id: hal-01479410 \\ https://hal.science/hal-01479410
}

Submitted on 24 Jun 2019

HAL is a multi-disciplinary open access archive for the deposit and dissemination of scientific research documents, whether they are published or not. The documents may come from teaching and research institutions in France or abroad, or from public or private research centers.
L'archive ouverte pluridisciplinaire HAL, est destinée au dépôt et à la diffusion de documents scientifiques de niveau recherche, publiés ou non, émanant des établissements d'enseignement et de recherche français ou étrangers, des laboratoires publics ou privés. 


\title{
Topology Optimization of a Reactionless Four-bar Linkage
}

\author{
S. Briot and A. Goldsztejn \\ Laboratoire des Sciences du Numérique de Nantes (LS2N), UMR CNRS 6004, \\ France, e-mail: \{Sebastien.Briot,Alexandre.Goldsztejn\}@ls2n.fr
}

\begin{abstract}
Most of existing works on the optimal design of balanced four-bar linkages deal essentially with the minimization of their inertia or input torques under balancing constraints. These approaches are not suitable to include constraints based on the elastic behavior of the mechanism. In order to solve this issue, we propose in this paper to perform the topology optimization of a reactionless four-bar linkage. Conditions for balancing the mechanism are first recalled and a topology optimization algorithm is run in order to maximize the first natural frequency while ensuring the balancing and constraining the mechanism compliance. We show that in order to obtain an interesting design solution, it is necessary to modify the balancing constraints in order to penalize them. Interesting design solutions are obtained in a rather short computational time.
\end{abstract}

Key words: Four-bar linkage, shaking force and shaking moment balancing, optimal design, topology optimization.

\section{Introduction}

Transmitting no reactions to the ground is very appealing in many applications such that space robotics or high-speed manipulation [10]. However, complete shaking force and shaking moment balancing is usually obtained by using both counterweights and counter-rotations, thus leading to an increase in the design complexity and to noise and backlash issues due to the use of gears [10].

In order to avoid the drawbacks in using counter-rotations, a reactionless four-bar linkage without them was proposed in [12]. For obtaining this property, conditions on both the geometry and the mass distribution of the linkage must be respected. It was later shown in [9] that this reactionless linkage can be used as an elementary block in order to design reactionless robots

In [10], the optimal design of the reactionless four-bar linkage was carried out for minimizing its input torques under balancing conditions constraints only. In this work, the shape of the links and counterweights is fixed and the authors focus on the optimal positioning of the counterweights. Other works deal with the optimal design of balanced four-bar linkages for allowing the full [8] or partial [5,7] dynamic balancing while optimizing energy consumption or input torque amplitude. More complete list of reference can be found in [1].

The main issue with the aforementioned methods comes from the fact that the shape of the links is already fixed (the design variables are their dimension) and deformation or vibration constraints may lead to an unfeasible design in practice (bulky mechanism to resist to the external efforts while ensuring the balancing conditions). For avoiding this issue, it is necessary to optimize the shape of the links. This was done in [5] for the partial balancing of the four-bar linkage, however the 


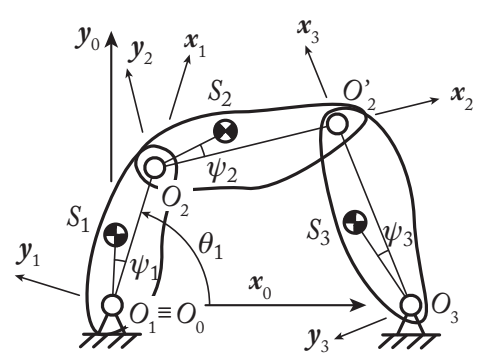

(a) General scheme

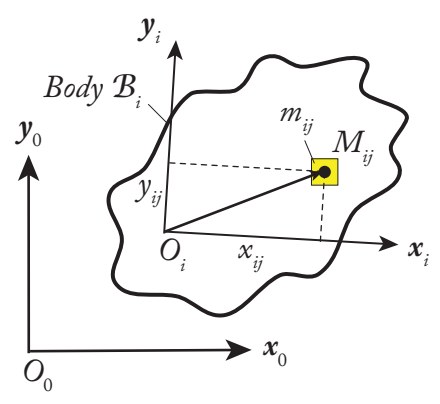

(b) Location of the finite element $i j$ in the body $\mathscr{B}_{i}$

Fig. 1 A general four-bar linkage.

approach proposed allows for finding only the external shape of the links. No voids can be included, which is not optimal w.r.t. the minimization of the link deformation while ensuring the decrease of the link mass.

Performing a Topology Optimization (TO) of the linkage can solve this issue [15]. This technique aims at optimizing the material distribution in a link in order to satisfy performance criteria including deformations or vibration constraints.

Our contribution in the present paper is to perform the TO of the reactionless four-bar linkage for ensuring its full balancing conditions while constraining elastic performance criteria. To the best of our knowledge, this is the first time that the TO of a fully force and moment balanced mechanism is performed. Furthermore, we show that balancing constraints must be partially penalized in order to obtain meaningful designs.

\section{Problem formulation}

The general scheme of the four-bar linkage is given in Fig. 1(a). In what follows,

- body $\mathscr{B}_{1}$ is the body between joints at $O_{1}$ and $O_{2}$, body $\mathscr{B}_{2}$ is the body between joints at $O_{2}$ and $O_{2}^{\prime}$ and body $\mathscr{B}_{3}$ is the body between joints at $O_{3}$ and $O_{2}^{\prime}$,

- $\ell_{1}$ is the distance between $O_{1}$ and $O_{2}, \ell_{2}$ is the distance between $O_{2}$ and $O_{2}^{\prime}$ and $\ell_{3}$ is the distance between $O_{3}$ and $O_{2}^{\prime}, \ell_{4}$ is the distance between $O_{1}$ and $O_{3}$,

- $r_{i}$ is the distance between $O_{i}$ and $S_{i}(i=1,2,3)$

- for body $i, S_{i}$ is the center of mass, $m_{i}$ is its mass, $z z_{i}$ is the moment of inertia expressed at $O_{i}, m x_{i}\left(m y_{i}\right.$, resp.) is the static moment around $\boldsymbol{x}_{i}\left(\boldsymbol{y}_{i}\right.$, resp.), i.e

$$
\left[\begin{array}{l}
m x_{i} \\
m y_{i}
\end{array}\right]=\int_{\mathscr{B}_{i}} \overrightarrow{O_{i} M_{i j}} \mathrm{~d} m=m_{i} r_{i}\left[\begin{array}{c}
\cos \psi_{i} \\
\sin \psi_{i}
\end{array}\right] .
$$

\subsection{Shaking force and shaking moment balancing conditions}

The conditions given in [12] for achieving the full shaking force and shaking moment balancing of the four-bar linkage without counter-rotations involve both contraints on the mechanism geometry and mass distribution. Three different set of 
links lengths are possible: Set 1: $\ell_{1}=\ell_{3}$ and $\ell_{2}=\ell_{4}$; Set 2: $\ell_{1}=\ell_{2}$ and $\ell_{3}=\ell_{4}$; Set 3: $\ell_{1}=\ell_{4}$ and $\ell_{2}=\ell_{3}$.

In what follows, we focus only on mechanisms designed with the first set of geometric constraints, which is the set most often studied in the papers (see for instance $[9,10])$ and corresponds to the anti-parallelogram linkage. In this case, the conditions on the mass distribution given in [12] for the full balancing can be rewritten as:

$$
\begin{gathered}
m y_{1}=0 \text { and } m y_{2}=0 \text { and } m y_{3}=0 \\
m x_{1} / \ell_{1}+m_{2}-m x_{2} / \ell_{2}=0 \text { and } m x_{3} / \ell_{3}+m x_{2} / \ell_{2}=0 \\
z z_{1}-m x_{1} \ell_{1}+z z_{2}-m x_{2} \ell_{2}=0 \text { and } z z_{3}-m x_{3} \ell_{3}+z z_{2}-m x_{2} \ell_{2}=0
\end{gathered}
$$

\subsection{Modeling of the linkage elastic behavior}

Topology optimization uses the same physical model as in the finite element method for modeling bodies and linkages: each body is meshed using finite elements. The presence or absence of an element $i j$ (i.e. the element $j$ of the body $\mathscr{B}_{i}$ ) is parameterized with a material density variable $\rho_{i j}$ which can take values between 0 (which represents the absence of material) and 1 (which represents the presence of material).

Based on these density variables, we use an interpolation scheme in order to define an artificial material. This method is called the Solid Isotropic Material with Penalization (SIMP, [2]) and is known to be the most effective and the most widely used material interpolation scheme. This interpolation scheme is adopted in order to avoid having optimization results with too much intermediate material density, i.e. in order to have a final design solution with densities only equal to $\rho_{i j}=1$ or $\rho_{i j}=0$ without too many intermediate values $\left(0<\rho_{i j}<1\right)$ that are difficult to manage by the designer.

The SIMP scheme is used to parameterize the Young's modulus associated with the stiffness matrix of the element $i j$ and it is defined as follows:

$$
E_{i j}=E_{\min }+\rho_{i j}^{p}\left(E_{0}-E_{\min }\right), \text { with } \rho_{i j} \in[0,1]
$$

where $E_{0}$ is the Young's modulus of the material, $E_{\min }$ is a very small stiffness value assigned to void regions in order to prevent the stiffness matrix from becoming singular, $p$ (typically $p=3$ ) is the penalization factor, and $E_{i j}$ is the Young's modulus of element $j$ of the body $i$ corresponding to the density variable $\rho_{i j}$.

Then, based on this definition of the Young's modulus for the element $i j$, we build its stiffness matrix. Finally, once all elementary matrices are defined, the computation of the body and linkage stiffness matrices is the same as in the traditional methodology [13].

The computation of the body and linkage mass matrices (necessary for the computation of the elastodynamic performance) is also based on the use of elementary mass matrices $\mathbf{M}_{i j}$ equal to

$$
\mathbf{M}_{i j}=\rho_{i j} \mathbf{M}_{i j}^{0}
$$


where $\mathbf{M}_{i j}^{0}$ is the mass matrix of the element $i j$ computed for a density $\rho_{i j}=1$.

Once the linkage stiffness and mass matrices are obtained, the elastic performance of the mechanism can be defined, such that the deformations or natural frequencies [13].

It should be mentioned that, in order to decrease the time for computing the elastic performance of the linkage, a Craig-Bampton model reduction technique [6] is applied on each body independently, as done in [4].

\subsection{Optimization problem}

The general formulation of a mono-objective TO problem is given by:

$$
\min _{\boldsymbol{\rho}} f(\boldsymbol{\rho}) \text { subject to } \rho \in[0,1]^{n}, \mathbf{g}(\boldsymbol{\rho}) \leq \mathbf{0}, \mathbf{h}(\boldsymbol{\rho})=\mathbf{0},
$$

where

- $\rho$ is the decision variable vector containing all element densities $\rho_{i j}$,

- $f, \mathbf{g}$ and $\mathbf{h}$ are functions of $\boldsymbol{\rho}$ characterizing performance indices or structure constraints.

Several optimization algorithms can be used, among which we can cite the Optimality Criteria method [17], the Method of Moving Asymptotes [16] or the Linearization Method [11] (LM) only recently used in the context of TO in [4]. We used the latter in this work. All these techniques have the same specificity: they require the symbolic computation of the functions and their gradients.

Here, we decided to maximize the first natural frequency for the linkage under the following constraints:

- the balancing equalities (2) to (4) must be respected. As shown in [4], the inertial parameters used in these equalities are all linear with respect to the decision variables in $\rho$.

- the compliance (i.e. twice the potential elastic energy or also the dot product of the nodal wrenches by the nodal displacements) must be lower than a given threshold under a given loading (as often done in TO, see for instance [15]).

For computing the compliance and natural frequency, we consider that the body $\mathscr{B}_{1}$ is actuated in $O_{1}$ and that the computation of these performances is made for $\theta_{1}=\pi / 2$ (Fig. 1(a)).

\section{Topology optimization}

\subsection{Initial domain}

The initial design domain for the proximal and distal links is represented in Figs. 2(a), 2(c) and 2(e). Four-bar geometric parameters are taken at $\ell_{1}=\ell_{3}=60 \mathrm{~mm}$ and $\ell_{2}=\ell_{4}=200 \mathrm{~mm}$. Each link has got two holes (voids) in which the joints will be inserted. Bodies $\mathscr{B}_{1}$ and $\mathscr{B}_{3}$ external shapes are rectangles of dimension $(150 \times 40) \mathrm{mm}$ and thickness of $10 \mathrm{~mm}$, while body $\mathscr{B}_{2}$ external shape is a rectangle of size $(220 \times 20) \mathrm{mm}$ and thickness of $1 \mathrm{~mm}$. For the meshing of the links, QUA4 finite elements (i.e. four-nodes rectangular planar elements) of size $1 \times 1 \mathrm{~mm}$. 
Elements associated with $\rho_{l} \mid$ Elements associated with $\rho_{r}$

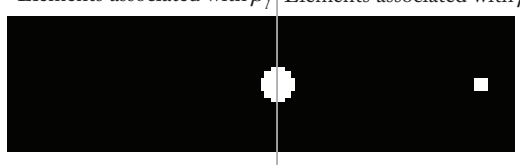

(a) Initial design domain for body $\mathscr{B}_{1}$

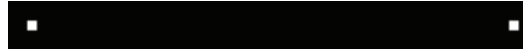

(c) Initial design domain for body $\mathscr{B}_{2}$

Elements associated with $\rho_{l} \mid$ Elements associated with $\rho_{r}$

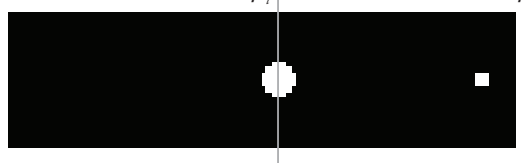

(e) Initial design domain for body $\mathscr{B}_{3}$

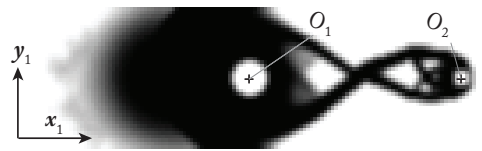

(b) First version of optimal design for body $\mathscr{B}_{1}$

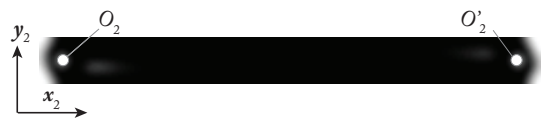

(d) First version of optimal design for body $\mathscr{B}_{2}$

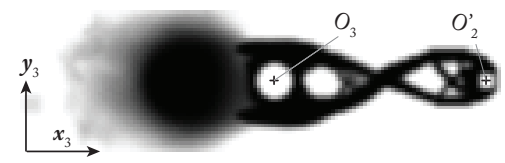

(f) First version of optimal design for body $\mathscr{B}_{3}$

Fig. 2 Initial design domain and first temptative optimal design for all bodies (black elements correspond to $\rho_{i j}=1$, white elements to $\rho_{i j}=0$, and gray elements to $0<\rho_{i j}<1$ )

Links are considered to be made of steel with Young's modulus $E_{0}=210 \mathrm{GPa}$, Poisson's ratio $v=0.3$ and density of $7800 \mathrm{~kg} / \mathrm{m}^{3}$. As a result, 6000 elements are used for meshing the bodies $\mathscr{B}_{1}$ and $\mathscr{B}_{3}$ while the body $\mathscr{B}_{2}$ is made of 4400 elements (Figs. 2(a), 2(c) and 2(e)).

\subsection{Optimization results}

We run the TO algorithm with a fixed threshold for the compliance value of 1.6. $10^{-3} \mathrm{Nm}$ for the assembled linkage under the following loading:

- at $O_{2}$ : a force of $15 \mathrm{~N}$ along $\boldsymbol{x}_{0}$, a force of $15 \mathrm{~N}$ along $\boldsymbol{y}_{0}$, a moment of $2 \mathrm{Nm}$ around $z_{0}$ on body $\mathscr{B}_{1}$, a moment of $1 \mathrm{Nm}$ around $z_{0}$ on body $\mathscr{B}_{2}$,

- at $O_{2}^{\prime}$ : a force of $15 \mathrm{~N}$ along $\boldsymbol{x}_{0}$, a force of $15 \mathrm{~N}$ along $\boldsymbol{y}_{0}$, a moment of $2 \mathrm{Nm}$ around $z_{0}$ on body $\mathscr{B}_{3}$, a moment of $1 \mathrm{Nm}$ around $z_{0}$ on body $\mathscr{B}_{2}$,

- at $O_{3}$ : a moment of $2 \mathrm{Nm}$ around $z_{0}$ on body $\mathscr{B}_{3}$.

As usually done in TO, in order to obtain a smoother layout without checkerboards problem, we modified the density variables assigned to the elements with the information of its neighborhoods as was proposed in [3].

All functions have been encoded with Matlab in the Windows 7 environment. The LM optimization algorithm is available under request to the second author.

The optimization process is run and we considered that the algorithm converged when the maximal change between two sequential iterations for any component of the density vector $\rho$ is lower than 0.01 . First results of optimization are shown in 


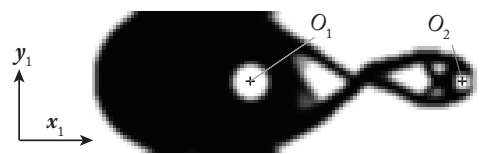

(a) Optimal design of body $\mathscr{B}_{1}$ : case 1

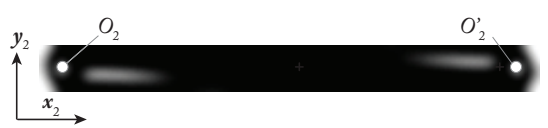

(c) Optimal design of body $\mathscr{B}_{2}$ : case 1

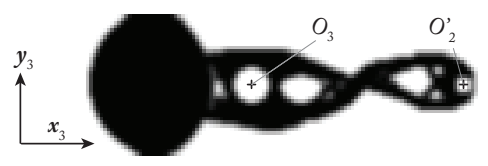

(e) Optimal design of body $\mathscr{B}_{3}$ : case 1

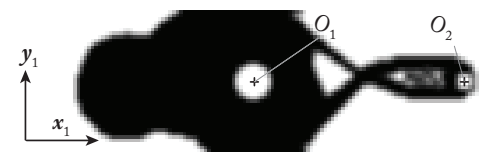

(b) Optimal design of body $\mathscr{B}_{1}$ : case 2

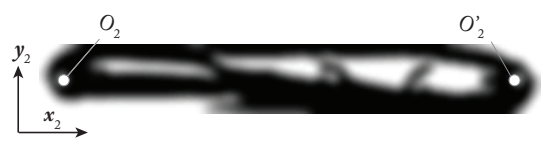

(d) Optimal design of body $\mathscr{B}_{2}$ : case 2

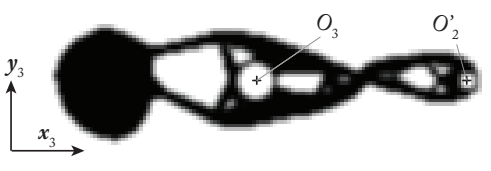

(f) Optimal design of body $\mathscr{B}_{3}$ : case 2

Fig. 3 Optimal design of bodies $\mathscr{B}_{1}, \mathscr{B}_{2}$ and $\mathscr{B}_{3}$ in two cases; case 1: the initial design domain of body $\mathscr{B}_{2}$ is the one depicted in Fig. 2; case 2: the initial design domain of body $\mathscr{B}_{2}$ is is changed: the body's height is now of $30 \mathrm{~mm}$ instead of $20 \mathrm{~mm}$ as in case 1 (black elements correspond to $\rho_{i j}=1$, white elements to $\rho_{i j}=0$, and gray elements to $0<\rho_{i j}<1$ )

Figs. 2(b), 2(d) and 2(f). The algorithm stopped after 524 iterations, with a maximal constraint violation of $5 \cdot 10^{-3} \%$. In totality, the optimization procedure took 28 minutes with a Pentium $42.70 \mathrm{GHz}, 16 \mathrm{~GB}$ of RAM. However, the algorithm had difficulty to converge (large oscillations in the value of the objective function, not displayed for reasons of page limitations) and finally attained a first natural frequency of $67 \mathrm{~Hz}$ (which is quite low, as will be shown below).

Obtained results showed that on the left-hand area of the points $O_{1}$ and $O_{3}$, the material density for bodies $\mathscr{B}_{1}$ and $\mathscr{B}_{3}$ is between 0 and 1 (gray elements), thus leading to bodies which are not easy to design by engineers [14]. We increased the number of allowed optimization iterations and obtained no improvement: These portions of materials are mostly here to fulfill the balancing constraints and have less impact on the compliance constraint or the frequency of the full linkage.

We propose here a way to improve this solution which is based on a partial penalization of the balancing constraint equations. As said in Sect. 2.3, the equality constraints (2), (3) and (4) are linear, i.e they can be written under the form:

$$
\mathbf{A} \boldsymbol{\rho}=\mathbf{A}_{l} \boldsymbol{\rho}_{l}+\mathbf{A}_{r} \boldsymbol{\rho}_{r}=\mathbf{0}
$$

in which $\rho_{l}$ contains the decision variables associated with the elements on the lefthand side of the gray lines in Figs. 2(a) and 2(e) for bodies $\mathscr{B}_{1}$ and $\mathscr{B}_{3}$ while $\rho_{r}$ contains all other variables, including those of the body $\mathscr{B}_{2}$. Thus, the vector $P_{l}$ contains the variables associated with the portions of materials which are almost here to fulfill the balancing constraints, which have few impacts on the linkage elastic performance, and which takes intermediary values for density.

\footnotetext{
${ }^{1}$ Constraints are normalized using their values computed for the initial design, except for Eq. (2) whose initial values are null.
} 
In order to force the values of the variables in $\rho_{l}$ to be only 0 or 1 , we modify the balancing equality (8) by raising the variables $\rho_{l}$ at the power of $q$ as follows:

$$
\mathbf{A}_{l} \rho_{l}^{q}+\mathbf{A}_{r} \rho_{r}=\mathbf{0}
$$

In our code, we put $q=2$ or 3 . To the best of our knowledge, this is the first time that penalization method is applied in order to achieve balancing constraints. Based on this new formulation, condition (9) does well represent the balancing equality (8) if all elements in $\rho_{l}$ are equal to 0 or 1 . Thanks to this penalization of the variables $\rho_{l}$, a small removal of material has a considerable impact on the balancing of the system, thus forcing the algorithm to impose 0 or 1 values to the variables $\rho_{l}$ in order to counterbalance the effect of the variables $\rho_{r}$. The optimization results by taking into account the constraints (9) instead of (8) are shown in Figs. 3(a), 3(c) and 3(e) (results obtained in 20 minutes, objective: first natural frequency of $646 \mathrm{~Hz}$, constraint violation of $1.4 \cdot 10^{-4} \%$ ). Results were obtained without any instability of the optimization algorithm and it can be observed that gray elements have been removed from the design solution.

Figures. 3(b), 3(d) and 3(f) show the same optimization problem but the difference comes from a change in the initial domain for body $\mathscr{B}_{2}$ which was increased (body's height is now of $30 \mathrm{~mm}$ instead of $20 \mathrm{~mm}$ ). Final objective was of $763 \mathrm{~Hz}$ and was attained in around 10 hours. It can be observed that a slight change in the design domain may lead to a totally different design solution.

\subsection{Discussion}

This work was made in order to show that TO can be used in order to obtain solutions in a rather short time of a complicated design problem which was to balance a fourbar linkage while ensuring deformation, compliance or frequency constraints.

However, in this paper, some issues have not been solved, which should be investigated later. First, the optimization is performed for compliance and frequency computed at $\theta_{1}=\pi / 2$. Thus, our optimization will be local by default. A more global optimization procedure ensuring the performance for any linkage configuration could be used (see [4] for instance) and some other optimization problems could be envisaged: objective and constraints could be changed.

It should be finally mentioned that our simulations have shown that the convergence of the algorithm is considerably sensitive by the threshold on the inequality constraints. In case all constraints are not achievable (i.e. there is no solution to the problem), the algorithm may become unstable.

\section{Conclusion}

Most of existing works on the optimal design of balanced four-bar linkages deal essentially with the minimization of their inertia or input torques under balancing constraints. These approaches are not suitable to include constraints based on the elastic behavior of the mechanism. In order to solve this issue, we performed in this paper the topology optimization of a reactionless four-bar linkage. 
In our paper, a topology optimization algorithm was run in order to maximize the first natural frequency while ensuring the balancing and constraining the mechanism compliance. We showed that in order to obtain an interesting design solution, it was necessary to modify the balancing constraints in order to penalize them. Interesting design solutions were obtained in a rather short computational time.

Future works include solving the problem in 3-D and also carrying out multimaterial topology optimization in order to ensure the balancing conditions by partially using materials with higher density leading thus to smaller mechanism footprint. The design of a prototype is also envisaged.

Acknowledgements This work was supported by the project RobEcolo funded by the French Région Pays de la Loire (Convention No. 2015-10773).

\section{References}

1. Arakelian, V., Briot, S.: Balancing of Linkages and Robot Manipulators - Advanced Methods with Illustrative Examples. Springer (2015). ISBN: 978-3-319-12489-6

2. Bendsoe, M., Sigmund, O.: Material interpolation schemes in topology optimization. Archive of Applied Mechanics 69 (1999)

3. Bourdin, B.: Filters in topology optimization. International Journal for Numerical Methods in Engineering 50 (2001)

4. Briot, S., Goldsztejn, A.: Global topology optimization of industrial robots with the linearization method. Mechanism and Machine Theory (2017). Under evaluation. Downloadable on http://www.irccyn.ec-nantes.fr/ briot/Download/BriotGoldsztejn.pdf

5. Chaudhary, K., Chaudhary, H.: Shape optimization of dynamically balanced planar four-bar mechanism. Procedia Computer Science 57, 519-526 (2015)

6. Craig, R.R., Bampton, M.C.C.: Coupling of substructures for dynamic analysis. AIAA Journal 6(7) (1968)

7. Erkaya, S.: Investigation of balancing problem for a planar mechanism using genetic algorithm. Journal of Mechanical Science and Technology 27(7), 2153-2160 (2013)

8. Farmani, M., Jaamialahmadi, A., Babaie, M.: Multiobjective optimization for force and moment balance of a four-bar linkage using evolutionary algorithms. Journal of Mechanical Science and Technology 25(12), 2971-2977 (2011)

9. Gosselin, C., Vollmer, F., Côté, G., Wu, Y.: Synthesis and design of reactionless three-degreeof-freedom parallel mechanisms. IEEE Transactions on Robotics and Automation 20(2), 191199 (2004)

10. Jiang, Q., Gosselin, C.: Dynamic optimization of reactionless four-bar linkages. Journal of Dynamic Systems, Measurement, and Control 132 (2010)

11. Pshenichnyj, B.: The Linearization Method for Constrained Optimization. Computational Mathematics. Springer (1994)

12. Ricard, R., Gosselin, C.: On the development of reactionless parallel manipulators. In: Proceedings of the ASME Design Engineering Technical Conferences and Computers and Information in Engineering Conference (IDETC/CIE 2000). Baltimore, Maryland (2000)

13. Shabana, A.: Dynamics of Multibody Systems. Cambridge University Press (2005)

14. Sigmund, O.: A 99 line topology optimization code written in MATLAB. Structural and Multidisciplinary Optimization 21(2), 120-127 (2001)

15. Sigmund, O., Maute, K.: Topology optimization approaches: A comparative review. Structural and Multidisciplinary Optimization 48(6), 1031-1055 (2013)

16. Svanberg, K.: The method of moving asymptotes - a new method for structural optimization. Numerical Methods in Engineering 24(2), 359-373 (1987)

17. Yin, L., Yang, W.: Optimality criteria method for topology optimization under multiple constraints. Computers and Structures 79(20-21), 1839-1850 (2001) 